\section{OCEANOGRAPHY \\ Bottom of the top of the world}

\author{
The floor of the Arctic Ocean \\ comes into sharper focus with the \\ publication of an improved version \\ of a bathymetric chart first released \\ in provisional form in 1999, and as \\ version 1 in 2001. Accurate mapping \\ of the ocean bottom is essential for \\ modelling deep ocean circulation, but \\ also has a political angle in defining \\ the extent of the continental shelf \\ - a serious consideration in such a \\ politically sensitive part of the world \\ as the Arctic. \\ The story behind the improved \\ bathymetric chart - IBCAO Version \\ 2.0 - is told by Martin Jakobsson \\ and colleagues in Geophysical \\ Research Letters (M. Jakobsson \\ et al. Geophys. Res. Lett. 35, L07602; \\ 2008). Its production is an \\ instructive case of new data being \\ married to a reinterpretation of old. \\ Most of the new data come from \\ mapping missions carried out \\ since 2000 with multibeam sonar
}

equipment aboard various vessels, including USCGC Healy, RV Polarstern and IB Oden. Multibeam sonar systems differ from the sidescan systems used, for example, to look at the shape of the sea floor or to detect wrecks, in providing information mainly about depth.

The more dramatic changes to version 2 over version 1 are that, as the authors laconically put it, the "deep abyssal plains are systematically ca. 50-60 m deeper ...". The revision stems from a metadata analysis of records collected by US Navy submarines over several decades, which are a central source of bathymetric information at high northern latitudes in particular. Conversion of data for version 1 was based on an assumption that the figure for the speed of sound in water used for the original calculations was $1,500 \mathrm{~m} \mathrm{~s}^{-1}$. But in many cases the figure applied

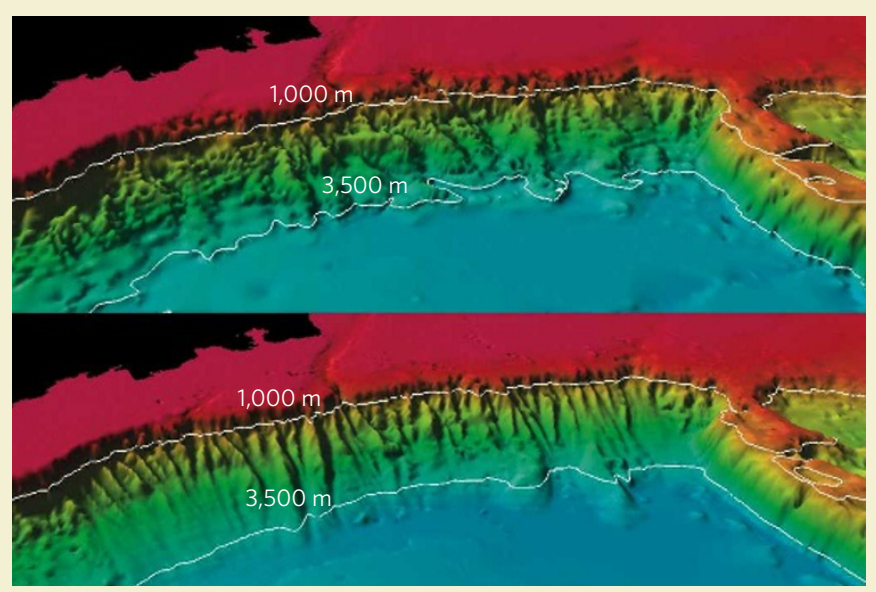

was $1,463 \mathrm{~m} \mathrm{~s}^{-1}$. Hence the change in estimated depth, which also helps to explain several anomalies evident in version 1.

The three-dimensional views shown here are depictions of the Alaskan Slope and Northwind Ridge before (upper image) and after Jakobsson and colleagues' exercise in producing version 2 . The image is about $650 \mathrm{~km}$ across, and the black area at the upper left is Alaska; the Northwind Ridge is the 'peninsula' on the right. The improved definition is evident in the sharper depiction of the gullies, caused by erosion, that scar the Alaskan Slope.

The new map is far from the final word. The authors point out that a near-perfect bathymetric model will require comprehensive multibeam coverage, which won't be available anytime soon. Meanwhile, more details on version 2 and derivations of it are available from www.ibcao.org. Tim Lincoln

\title{
ASTROPHYSICS
}

\section{Exhaust inspection}

\section{David L. Meier}

\section{What do you see if you peer into the exhaust of a jet engine larger than our Solar System? Only astronomers with the largest radio telescopes can see the full picture - and definitive observations are beginning to filter through.}

Cosmic jets - enormously energetic, highly directed beams of charged particles - can be ejected by stars as they form ${ }^{1}$, as they $\mathrm{die}^{2-4}$, and even, in certain instances, as they are reborn, rekindled by the accretion of surrounding gas ${ }^{5,6}$. The energy carried away by a jet can help to bring a dying massive star to explosion in a supernova, or disperse a less massive one as a planetary nebula. Jets provide some of the heating of the gas between stars, and can even disrupt the intergalactic medium in galaxy clusters.

Despite a profusion of hypotheses for how these cosmic power generators work, astronomers still don't have a clinching theory, largely because of a dearth of observations with which to compare models. New findings from Marscher et al. (page 966 of this issue) $)^{7}$ go a long way to redressing this imbalance. The authors report how, in unprecedented detail, they imaged a jet engine belching out a puff of exhaust, allowing real-time insight into how jets work.

Theories exploring the role of gas accretion in jet acceleration originated in the late 1970s and early $1980 \mathrm{~s}^{8-10}$, and have more recently been extended to take account of features such as flows at close to the speed of light and the very hot temperatures of the jet plasma ${ }^{11}$. Many of the jet-forming objects are dense, accreting agglomerations of matter such as neutron stars or black holes, both of which originate from failed stars. But jets are also produced by objects such as fading 'white dwarf' stars, or even stars such as the Sun when they are first born. Understanding jet emission from one type of these objects, therefore, might lead to an understanding of many others.

The favoured picture that has emerged is of a mechanism similar to the operation of a fighter plane's jet engine, but confined within the lines of a strong magnetic field rather than a steel casing. Rotation of the magnetosphere around the central object creates a stiff, helical field that acts as both a turbine and a nozzle, expelling the exhaust flow and collimating it into a narrow beam (see Fig. 3 on page 968). Far from the object, this natural pinching action can overcollimate the beam, so that it starts to converge. The point of maximum convergence is known as the modified magnetohydrodynamic fast point; beyond that point, the jet can produce a strong 'fast-mode' shock wave that can distort or destroy the well-ordered structure of the magnetic nozzle. Other, less dramatic, 'slow-mode' shocks - pressure waves that travel parallel to the twisting field lines and do not disrupt them - can develop before the flow reaches the modified fast point.

The equations governing these processes are very complex, and several simplifying assumptions are needed to solve them. For that reason, the whole picture of magnetic jet acceleration just sketched is still a matter of debate. Marscher et al. ${ }^{7}$ are the first to test the picture observationally. Their results, at least partially, confirm the model.

The authors' test-bed was the notoriously unruly BL Lacertae, the archetype of a particularly extreme form of jet emitter known as a blazar. BL Lacertae is an active galaxy containing a central supermassive black hole, and produces a jet with a speed $99 \%$ that of light. The jet, to within $10^{\circ}$, is pointing directly at us, so that looking down into the jet nozzle allows Earth-bound observers to examine the engine's inner workings directly.

This particular jet emits enormous amounts of energy at radio frequencies, and is huge - about two light years across. So, despite its immense distance from us (about 900 million light years), its angular size seen from Earth is fairly large, at around 0.5 milliarcseconds 